\title{
Electromagnetic Diffraction by Fractal Dusts, Triangles and Carpets: A Kirchhoff Approach to Circulation
}

\author{
James M. Christian, Holly A. J. Middleton-Spencer \\ Joule Physics Laboratory, University of Salford, Greater Manchester M5 4WT, United Kingdom
}

The diffraction of plane waves by perfectly-conducting thin screens is of fundamental physical and mathematical interest in electromagnetics [1]. Classic laser-optics experiments involve both open (single- and double-slit arrangements) and closed (circular and regular-polygon) apertures, with analyses often being confined to the Fresnel [2] and Fraunhofer [3] regimes. Here, we consider a class of scattering problem involving fully-2D fractal screens, where the scatterer possesses the property of self-similarity. A more general formulation of the diffracted wave, based on Kirchhoff's theory and 3D Green's functions [4], is also deployed.

Our attention focuses on three well-known fractal shapes constructed from initiator-generator algorithms: the Cantor dust, and the triangle (see Fig. 1, top row) and carpet devised by Sierpinski [5]. The initiator may be regarded as a 'hole' contained inside an otherwise-opaque plane, or as an opaque obstacle that is surrounded by otherwise-empty space. These two distinct configurations-which we define as a screen and its complement, respectively - are related to one another in quite a subtle way [6]. Moreover, stage $n=0,1,2, \ldots$ comprises a finite number of identical and suitably scaled regular-polygon subdomains (e.g. for the Sierpinski triangle, one has a set of $3^{n}$ elements, each of whose area is $1 / 4^{n}$ times that of the initiator). The fully-developed fractal shape in each case also has a Lebesque measure of zero, which we interpret physically as the zero-area limit.
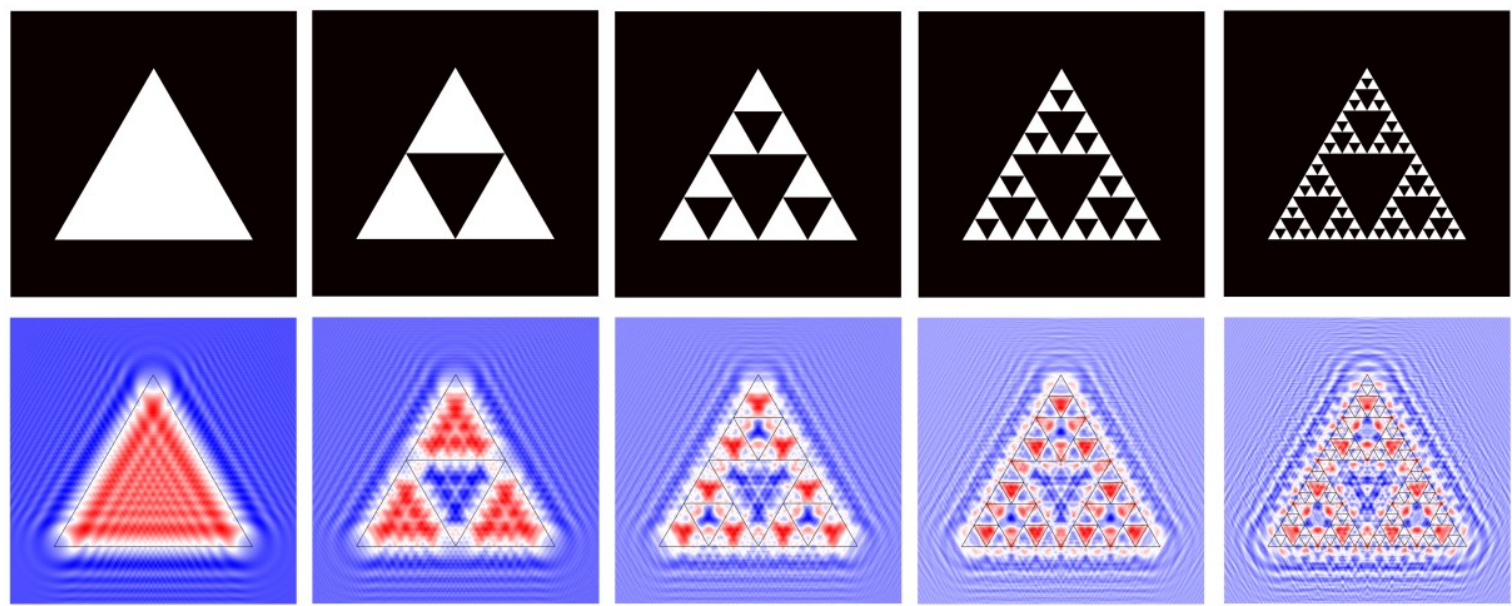

Fig. 1 Top row: Initial $(n=0)$ and first four pre-fractal levels $(n=1-4)$ of the Sierpinski triangle, which emerges in the limit $n \rightarrow \infty$ and has a Hausdorff dimension of $D=\log (3) / \log (2) \approx 1.58$ [5]. Bottom row: Snapshot in time of diffracted waves behind the screen (black lines mark the geometrical projection of the subdomain boundaries). The initial triangle is contained within a circle of radius $1 \mathrm{~mm}$, and the illuminating wavelength is set to $539 \mathrm{~nm}$ (corresponding to green light).

In this presentation, we will show how the fractal-screens problem may be addressed by solving the scalar Helmholtz equation in 3D. As a general strategy, the standard integration over the entire aperture domain is transformed (without approximation) into a circulation [7] around the constituent sub-domain boundaries. This theoretical device proves to be an enormously powerful tool for facilitating computations on pre-fractal shapes, particularly those involving non-orthogonal edges (see Fig. 1, bottom row). Boundary conditions (BCs) play a key role in determining uniquely the electric field in the surrounding space. Following Kirchhoff [4], we let the diffracted wave and its normal derivative vanish on the screen while, in the 'hole' regions, their incident planewave values are assumed. Fresnel and Fraunhofer formulations (both of which are largely insensitive to the details of the BCs) emerge asymptotically, as required. We conclude by discussing the strengths and weaknesses of the Kirchhoff representation - particularly in the fractal-domains context—and pose some open questions.

\section{References}

[1] J. D. Jackson, Classical Electrodynamics, $3^{\text {rd }}$ ed. (John Wiley, New York, 1999).

[2] J. G. Huang, J. M. Christian, and G. S. McDonald, "Fresnel Diffraction and Fractal Patterns from Polygonal Apertures," J. Opt. Soc. Am. A 23, 2768 (2006).

[3] R. C. Smith and J. S. Marsh, "Diffraction Patterns of Simple Apertures,” J. Opt. Soc. Am. 64, 798 (1974).

[4] G. Barton, Elements of Green's Functions and Propagation: Potentials, Diffusion and Waves, (Oxford, Clarendon Press, 2005).

[5] K. Falconer, Fractal Geometry: Mathematical Foundations and Applications, $2^{\text {nd }}$ ed., (John Wiley, Chichester, 2003).

[6] S. N. Chandler-Wilde and D. P. Hewett, "Well-Posed PDE and Integral Equation Formulations for Scattering by Fractal Screens," SIAM J. Math. Anal. 50, 677 (2018).

[7] J. S. Asvestas, "Line Integrals and Physical Optics. Part II. The Conversion of the Kirchhoff Surface Integral to a Line Integral," J. Opt. Soc. Am. A 2, 896 (1985). 